\title{
BMJ Open Storytelling as a research tool and intervention around public health perceptions and behaviour: a protocol for a systematic narrative review
}

\author{
Becky McCall, ${ }^{1}$ Laura Shallcross, ${ }^{1}$ Michael Wilson, ${ }^{2}$ Christopher Fuller, ${ }^{3}$ \\ Andrew Hayward ${ }^{4}$
}

To cite: McCall B, Shallcross L, Wilson M, et al. Storytelling as a research tool and intervention around public health perceptions and behaviour: a protocol for a systematic narrative review. BMJ Open 2019;9:e030597. doi:10.1136/ bmjopen-2019-030597

- Prepublication history for this paper is available online. To view these files, please visit the journal online (http://dx.doi. org/10.1136/bmjopen-2019030597).

Received 21 March 2019 Revised 26 August 2019 Accepted 16 October 2019

Check for updates

(C) Author(s) (or their employer(s)) 2019. Re-use permitted under CC BY-NC. No commercial re-use. See rights and permissions. Published by BMJ.

${ }^{1}$ Institute of Health Informatics, University College London,

London, UK

${ }^{2}$ School of the Arts, English and Drama, University of Loughborough, Loughborough, UK

${ }^{3}$ Institute of Health Informatics, UCL, London, UK

${ }^{4}$ Institute of Epidemiology and Health, University College London, London, UK

Correspondence to

Becky McCall;

becky.mccall.18@ucl.ac.uk

\section{ABSTRACT}

Introduction There is a growing trend to use storytelling as a research tool to extract information and/or as an intervention to effect change in the public knowledge, attitudes and behaviour (KAB) in relation to public health issues, primarily those with a strong element of disease prevention. However, evidence of its use in either or both capacities is limited. This protocol proposes a systematic narrative review of peer-reviewed, published literature on the use of storytelling as a research tool within the public health arena.

Methods and analysis Medline, EMBASE, PsycINFO, ERIC (Educational Resources Information Center), Web of Science, Art and Humanities database (ProQuest), Scopus and Google Scholar will be searched for studies that look at the use of storytelling in the research of pressing current public health issues, for example, vaccinations, antimicrobial resistance, climate change and cancer screening. The review will synthesise evidence of how storytelling is used as a research tool to (a) gain insights into $K A B$ and (b) to effect change in $K A B$ when used as an intervention. Included studies will be selected according to carefully defined criteria relevant to public health issues of interest, and data from qualitative, quantitative and mixed-methods studies will be extracted with a customised data extraction form. A narrative synthesis will be performed according to Economic and Social Research Council guidance from Popay, J, 2006. The study protocol follows the recommendations by the Preferred Reporting Items for Systematic Review and Meta-Analysis Protocols (PRISMA-P).

Ethics and dissemination Formal ethical approval is not required for this study, as no primary data will be collected. Dissemination will involve publishing results of this study in relevant peer-reviewed journal(s). Where possible, the study results will also be presented as posters or talks at relevant medical conferences and meetings.

PROSPERO registration number CRD42019124704

\section{INTRODUCTION}

Stories and storytelling help us to make sense of our thoughts and experiences, our interactions with the environment and each other, to formulate our beliefs, our identities and our values. ${ }^{1}$ Most poignantly, the making of
Strengths and limitations of this study

- The review will provide information on how storytelling has been used in important public health issues, for example, climate change, vaccination and cancer screening.

- The review will inform further use of storytelling in these and other public health issues, in particular antimicrobial resistance, to gain insight on public perceptions, and to communicate and disseminate information, and to potentially effect change in relevant behaviours.

- This study protocol follows the recommendations by the Preferred Reporting Items for Systematic Review and Meta-Analysis Protocols.

- The selection of studies, and data extraction will use systematic review management software (SUMARI; Joanna Briggs Institute, Australia), and critical appraisal will use the QATSDD quality assessment tool, developed by Sirriyeh, R and colleagues, 2012, which is suitable for the quality assessment of qualitative, quantitative and mixed-methods studies. The review will be conducted by two independent authors.

- Studies included following this review protocol are unlikely to be homogeneous in methods limiting the ability to draw reliable conclusions and generalisations.

stories 'reveals things to us that we know but didn't know we knew', according to phenomenological philosopher Maurice MerleauPonty in $1964 .^{2}$

Essential to storytelling is that it seeks to convey an experience in such a way that it seems real. ${ }^{3}$ There is appeal in storytelling because it often presents information incorporated within a personal account that engages the reader and may validate their own experiences. ${ }^{4}$

A story is often loosely defined as having a beginning, a middle and an end, with a protagonist (often human), an object, a 
practice or an idea, followed by a form of transformation or conflict. ${ }^{5}$ Throughout the relevant literature, the term 'narrative' is often used interchangeably with 'story'. However, the events that comprise a certain story can be presented in many different ways forming different narratives, chronologically or not, but the story remains the same. Reshuffling the order of events changes the narrative, not the story. ${ }^{6}$ The terms 'story' and 'storytelling' will be preferred in this review, unless specific cases require the use of 'narrative'.

\section{Storytelling as a research method}

This review seeks evidence of peer-reviewed studies that use storytelling as a research method or tool and relates to people telling their personal stories of real-life or authentic experiences around public health issues.

The process of storytelling has multiple research aims. Included in these aims is its ability to inform the researcher (through extraction of information), but also as an intervention to facilitate a process of 'reflection and reworking of experience and knowledge in the research participant'. ${ }^{7}$ Storytelling has been used as a tool to gain insight into public knowledge, attitudes and behaviour $(\mathrm{KAB})$, for example, storytelling has been used with African-American women who fail to attend breast cancer screening and has revealed attitudes and behaviour driven by pain, fear, loss and faith in God. The same research also used storytelling to clarify misinformation, validate personal experiences and enhance learning around the importance of screening. ${ }^{8}$

It needs to be noted that unlike more conventional qualitative research methods, storytelling is an emergent research method and validation remains to be established. However, lack of a substantial body of validation does not justify dismissing storytelling as a research method. Ideally, this review will identify studies that provide evidence of the validity of storytelling as used in the research context.

Storytelling has been used as a research method in various disciplines, some of which touch on public health, some of which are removed from it. For example, social work, ${ }^{9} 10$ healthcare and its delivery, ${ }^{11}$ understanding marginalised communities ${ }^{12}$ and anthropology, ${ }^{13}$ to provide a few examples.

In attempting a definition of storytelling as a research tool, first, there is a distinction to be made between science and storytelling/narrative as two research paradigms. Second, storytelling needs to be distinguished from other forms of narrative research, for example, narrative medicine. According to Bleakley, science and narrative are two ways of knowing. Bleakley points out the value of story compared with more conventionally analytical methods that 'tend to lose the concrete story and its emotional impact to abstract categorisations, which may claim explanatory value but often remain descriptive'. Essentially, within clinical education at least, Bleakley refers to how narrative offers value over and above objective measures, pointing out that while objective morbidity and mortality data characteristically remain faceless, narrative inquiry often seeks to personalise and also to engage proactively with its research population through deliberate intervention, as research with, not on, people. ${ }^{14}$

Reflecting Bleakley, this systematic review aims to find examples of where research has been carried out with people (within the context of various public health topics) rather than on people, and work that seeks to engage proactively with the study population via storytelling as a method.

Storytelling as a research method shares some similarities but also notable differences to narrative medicine as a research method. According to Columbia Narrative Medicine, Columbia University Irving Medical Center, 'narrative medicine fortifies clinical practice with the narrative competence to recognize, absorb, metabolize, interpret, and be moved by the stories of illness' ${ }^{15}$ Physician and advocate of narrative medicine, Rita Charon, in a paper in JAMA, describes narrative medicine as, 'Medicine practiced with narrative competence,.. is proposed as a model for humane and effective medical practice'. ${ }^{16}$ Narrative medicine appears to be more closely aligned with physicians' practice and the patient-physician relationship (aimed at improving patient care) than the storytelling research method that this systematic review aims to explore. This review aims to seek data on KAB from members of the public and does not serve the purpose of improving a physicianpatient relationship or improving care as directly as narrative medicine appears to do.

In practice, storytelling as a tool in research might adopt various formats. One that has found prominence in recent years, with an emergent literature base generally, as well as in the field of health research, is digital storytelling (DST) comprising a 25 min video ${ }^{17}$, a 3-5 min short video, ${ }^{18}$ 'Photovoice' (photo collections to promote dialogue) ${ }^{19}$ or verbal telling of personal stories. ${ }^{80}$ DST has the potential to capture lived experiences and share research findings in a manner that is highly engaging and possibly made accessible on a digital platform. ${ }^{21}$

\section{Storytelling as a research tool alongside conventional qualitative research methods}

Storytelling as a qualitative research method is still an emergent area and may serve to complement data sourced via more conventional, empirical qualitative research methods. However, certain nuances of individuals' insights associated with their experiences might not be accessible via some of these more established methods of inquiry.

Moreover, stories do not reveal one, single discoverable truth because truth is a matter of degree and perspective. In this respect, using the telling of a story as a research tool rests on a premise that is starkly different to that of a conventional scientific method. ${ }^{5}$ Both the established scientific method and the 
storytelling research method each provide a distinctive way of ordering experience and constructing reality, and using the two knowledge systems to complement and enhance each other might provide broader and more in-depth insight into an experience than using one method alone.

In a discussion paper by Dahlstrom, the author addresses storytelling as a means to communicate science to non-expert audiences. According to Dahlstrom, narratives are easier to comprehend, and audiences find them more engaging than traditional logical-scientific communication. The nature of learning from storytelling differs from that derived from more conventional scientific information, for example, from statistical data. Scientific information provides abstract truths that can be applied to a specific case, as in deductive reasoning, whereas narrative information follows inductive reasoning, which often involves a depiction of an individual experience from which an inference to a general or even a collective truth can be made. ${ }^{22}$

Storytelling is a highly nuanced means of communication, usually articulating cause-and-effect relationships between events over a period of time, and often in relation to a certain character. ${ }^{16}$ It is also grounded in a level of realism that might be less evident with other forms of communication. Storytelling potentially draws on commonalities between the story or the storyteller and the listener or reader. This, combined with the underlying assumption of credibility in the teller's story or experience, can potentially motivate and persuade individuals towards behavioural change and reduces resistance to any action implied by the message. ${ }^{23}$

Studies have used stories and storytelling for their value in both communicating with and influencing others. Among the reasons for choosing storytelling as a research tool, one of the most important is that it is a highly accessible modality that does not require specialised knowledge and skills to connect with, or derive meaning from. ${ }^{24}$

\section{Storytelling to change KAB in public health}

Whether smoking cessation, obesity, health-related climate change or cancer screening, many of the key issues in public health today require the sharing of information in a meaningful way that resonates with the receiver and triggers a positive change in knowledge, attitudes and ultimately behaviours. The lay public largely sources its information on scientific matters in narrative format, for example, from mass media, which relies on storytelling to optimise engagement with the reader, listener or viewer. ${ }^{22}$ In addition, most health-related knowledge and/or evidence is largely objective, often referring to statistics and appeals to logic and reason to support a certain practice or health-related behavioural change. However, there is a growing movement towards other forms of health communication including storytelling. ${ }^{25}$

The list of public health issues that might lend themselves to storytelling as a research tool is extensive, but of key interest in this review is any public health issue that bears a personal cost in the immediate term but potentially provides a wide-scale health benefit on a population level in the longer term. Vaccination, climate change, antimicrobial resistance (AMR) and cancer screening all provide typical examples.

Of particular interest to the authors of this review is any public health issue that closely reflects key features of AMR because future research aims to focus on this topic.

Regarding AMR and antibiotic use, a 2018 report by the Organisation for Economic Cooperation and Development estimated that AMR could cause approximately 2.4 million deaths in Europe, North America and Australia by 2050 if effective control measures are not taken. ${ }^{26}$ AMR needs to be addressed on multiple fronts, but of interest here is the public perception of AMR and how a more nuanced understanding of this, potentially sourced via storytelling, might help to change public perceptions and practices towards antibiotic use and AMR. In 2009, Edgar et al note that 'This [antibiotic resistance] is not a problem that will go away without a concerted effort to change the beliefs, attitudes and behaviour of key populations'. ${ }^{27}$

Various studies shed light on the need for more, and alternative research methods to investigate public understanding of AMR and antibiotic use. ${ }^{28}{ }^{29}$ Storytelling might reveal insights not necessarily obtainable via interviews and focus groups.

Potential research would use the storytelling approach as an alternative to, or to complement other more conventional qualitative research methods, to investigate public perceptions of the issues at stake, and to potentially develop a storytelling intervention to improve understanding and behaviours.

\section{Rationale for the review}

The rationale for this systematic narrative review rests on the premise that storytelling may have value as a qualitative research method used in the context of a public health issue with global impact.

To explore this, the review will involve two stages aimed at arriving at the most relevant studies for full-text review. Stage 1 will involve a search that will be wider in the scope of public health issues included than in stage 2, and will involve basic quantification of peer-reviewed studies on the use of storytelling as a research and/or an interventional tool in public health. Stage 2 will involve the careful selection of topics that will enter into later stages of the review. Topics will be selected based on certain criteria outlined in table 1, but essentially, topics of interest will be public health issues with a strong preventative element that involve a personal cost in the immediate term but population-wide gain in the long term.

This systematic narrative review proposes to explore storytelling as a means of sourcing data to uncover public $\mathrm{KAB}$ through gathering, analysing and critiquing, as well as to explore storytelling as an intervention used in the research context to effect change in $\mathrm{KAB}$. 


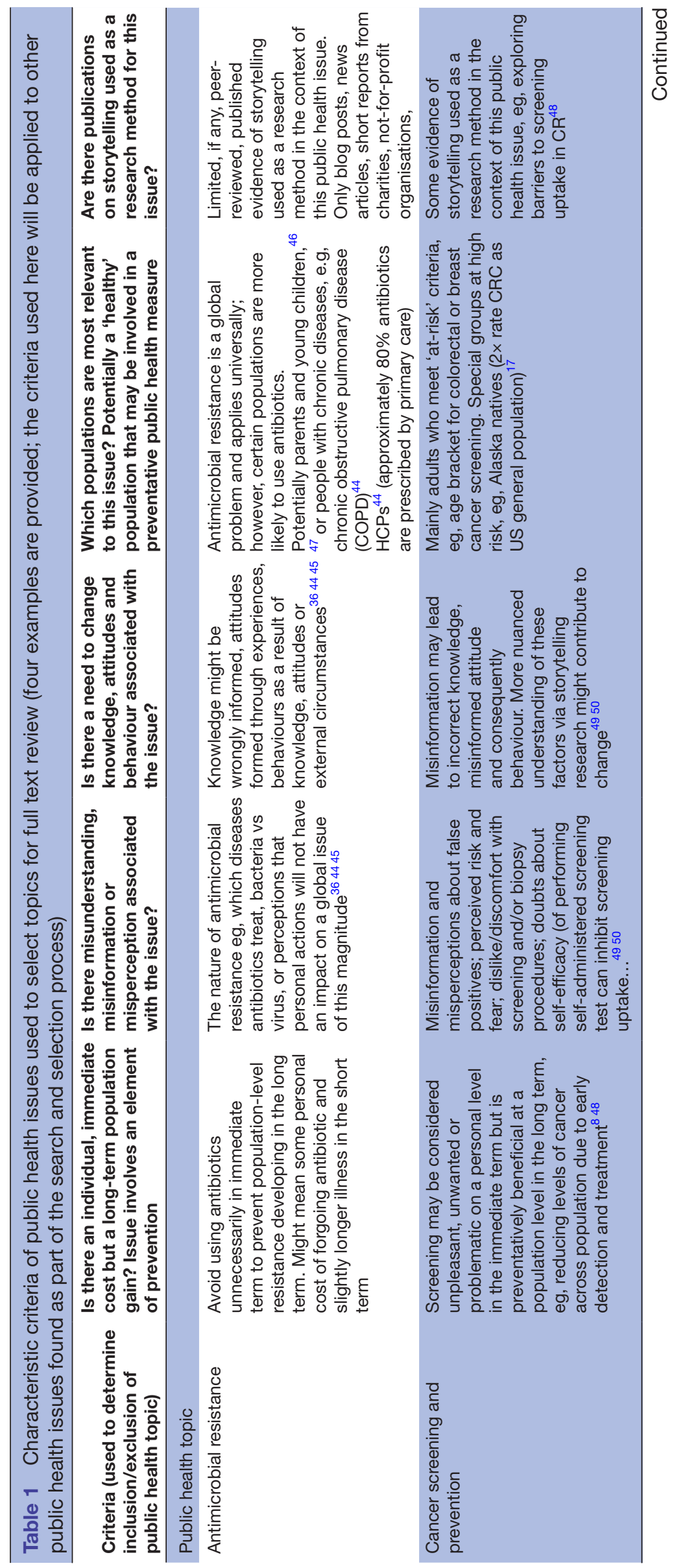




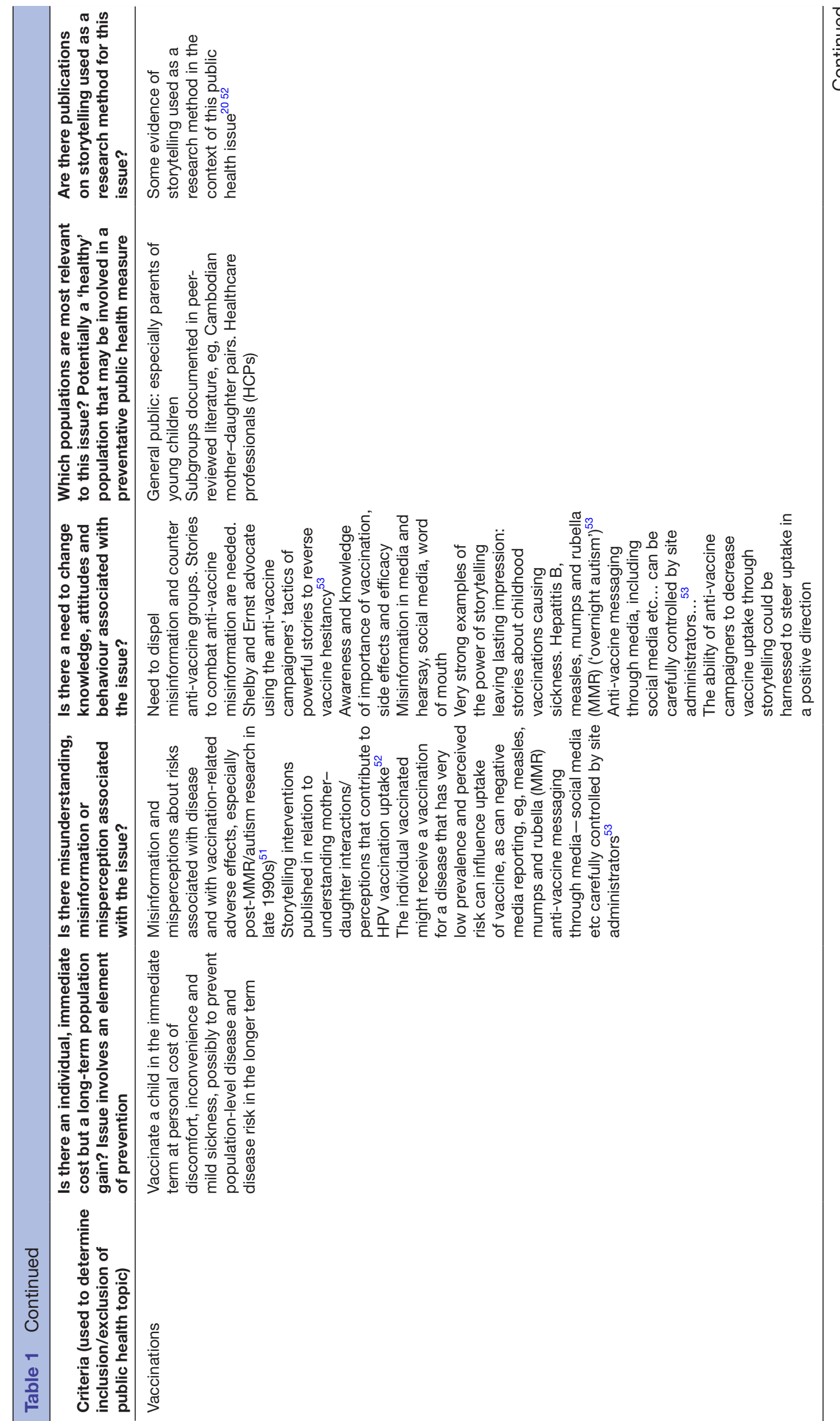




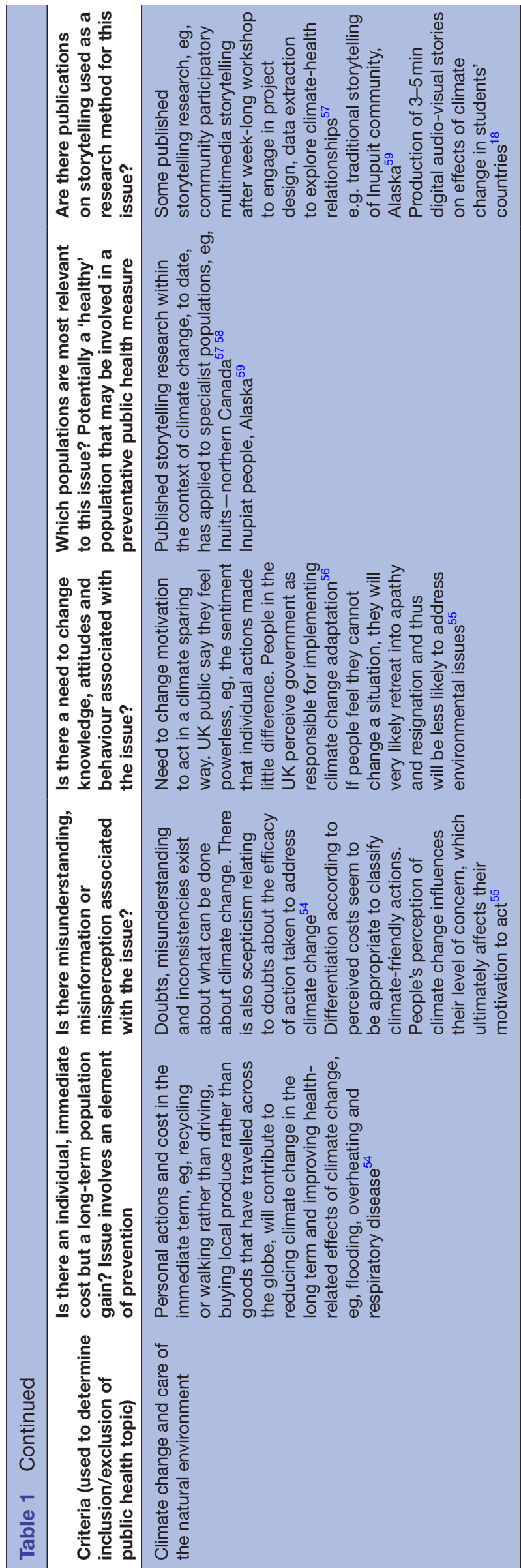

Aim

To synthesise studies that use storytelling as a research tool for understanding and/or influencing public opinion on public health/prevention issues.

\section{Objectives}

This systematic narrative review aims to

- Quantify the distribution of peer-reviewed, published studies that use storytelling as a research tool according to the public health issue.

- Use narrative synthesis to report on studies that employ storytelling as (a) a research tool to gain insight into $\mathrm{KAB}$, and/or (b) an intervention to effect change in $\mathrm{KAB}$.

- Determine the nature and value of storytelling by

- Describing the nature of, and the value of information obtained via storytelling,

- Determining to what extent storytelling can uncover the barriers and facilitators (mis/information, mis/beliefs, mis/understanding) that underlie perceptions and behaviours relating to certain public health issues, and to what extent storytelling as an intervention may effect change in these respects,

- Determining the impact and validity of storytelling as a research tool to source information, and/or to engage and communicate public health messages to effect change,

- Comparing data obtained via storytelling to other qualitative and quantitative methods, if these data are available.

- To gain understanding of how to run effective storytelling workshops with a view to guiding future research.

\section{Research question}

In accordance with the aim and objectives, the following question will guide the project:

What evidence supports the use of storytelling as a research method: (a) to gain insight on public $\mathrm{KAB}$, and (b) as an intervention to effect change in public KAB, in relation to issues of public health?

\section{Methods}

This systematic narrative review will follow a comprehensive process using rigorous methodological guidelines to synthesise the diverse forms of research evidence found (different public health issues; qualitative, quantitative and mixed-methods studies). The recommendations by the Preferred Reporting Items for Systematic Review and MetaAnalysis Protocols (PRISMA-P) 2015 will be followed. ${ }^{30}$

The study started in June 2019 and the anticipated completion date is January 2020.

\section{PATIENT AND PUBLIC INVOLVEMENT}

Patients or public were not involved in the development of this protocol. 


\section{Inclusion and exclusion criteria (eligibility of studies)}

Study design

Qualitative, quantitative and mixed-methods peerreviewed primary studies that use storytelling within the context of research will be included.

Studies should include storytelling used as a research tool to extract information on $\mathrm{KAB}$ relating to any chosen public health topic (part ' $a$ ' of the research question) whether or not they formally seek to measure the validity of storytelling; and/or studies that use storytelling as an intervention aimed at effecting change in $\mathrm{KAB}$ whether or not they formally evaluate the impact on these outcomes (part 'b' of the research question).

Studies should be included if the stories are told by individuals, for example, as a community participation project that seeks storytelling of personal experiences as opposed to stories related via the media (print, online, broadcast or other media including social media). Personal stories, as told by the individual or someone close to the individual who is central to the experience being related, are sought, rather than stories that are told in a journalistic or reporting setting.

Studies that formulate a story or stories based on an integration of the findings of numerous interviews will be excluded-such studies are not first-hand, direct, personal experiences. Also, any studies that discuss storytelling or take a review format will be excluded.

Studies will most likely include participants who are members of the general public, often belonging to a defined subgroup, and/or patients. Storytelling will be shared between participants and the researcher(s).

\section{Subject matter}

According to the WHO definition, public health comprises the 'art and science of preventing disease, prolonging life and promoting health through the organized efforts of society'. ${ }^{31}$ In this review, any public health topic that satisfies this definition but that also satisfies most, if not all, criteria listed in table 1 will be selected for full-text review. In particular, studies should have an element of prevention, for example, prevention of AMR, prevention of HIV/AIDs, prevention of climate change, prevention of future cancers or prevention of vaccinepreventable diseases. Studies should also incur some personal cost, for example, forgoing the opportunity to shorten your illness through avoidance of antibiotics, risk of adverse consequences such as severe disease following a mild infection, screening-related harm, vaccine side effects, aversion to use of condoms or inconvenience of recycling.

Studies will be excluded if they are primarily clinical in nature rather than public health related, or if they do not relate to either enhanced understanding or seek to change $\mathrm{KAB}$. They will also be excluded if the public health topic addressed does not have a preventative element nor incur some personal cost in the immediate or short term.
Studies will also be excluded if they apply to highly specific population subgroups, findings of which cannot reasonably be used to guide future research.

\section{Language}

The systematic review will be restricted to Englishlanguage studies only. An unrestricted scoping review suggests no evidence that limiting to English language only would be associated with bias.

\section{Dates}

Dates will include studies from 1990 to the present, which is a deliberately wide period of time due to the anticipated limited number of peer-reviewed and relevant reported studies available. Also, storytelling as a research tool is a relatively emergent area of research, so most papers are likely to have been published since the year 2000 .

\section{Demographics}

Again, due to the anticipated limited number of relevant studies in the emergent field of storytelling in public health, studies will include populations of all age ranges and demographic backgrounds.

\section{Types of interventions}

Storytelling or digital storytelling as a research tool to understand and/or effect change in $\mathrm{KAB}$ towards the public health issue of interest.

\section{Contexts}

Climate change

Vaccination

$\mathrm{HIV} / \mathrm{AIDS}$

Cancer screening

Sexual health

Mental health

Other public health topics as found on the search

Note that not all contexts will proceed to stage 2 of the selection process if the specified criteria are not met, at least in most part.

\section{Outcome measures}

Primary outcomes

Within the context of the public health issues selected for review, the following outcomes will be explored:

- Characteristics of the stories featured in the study, including

- The protagonist: a personal story, a story about others or a collective community story;

- The vehicle for storytelling: written, verbal, visual, audio-visual, other;

- The narrator: first or third person;

- Any other relevant characteristics.

- Specific aims and methods used in the studies in relation to

- Storytelling used as a research tool to extract information including information on $\mathrm{KAB}$,

- Storytelling used to effect change in KAB when used as an intervention. 
- Key insights, impacts and other evidence to support the use of storytelling to

- Gain insight into KAB relating to the public health issue of interest,

- Effect change in KAB relating to the public health issue of interest (eg, the dispelling of misinformation, knowledge acquisition or change, or change in beliefs, attitudes and practices).

\section{Secondary outcomes}

Evidence to support the 'validity' or, in broad terms, the 'value' and 'appropriateness' of storytelling as a means of drawing out nuanced information on the public's experiences, and of effecting change or having an impact on $\mathrm{KAB}^{32}$

\section{Information sources}

\section{Electronic searches}

To capture all relevant studies, the search will refer to the following databases. To maximise the return of relevant articles, numerous databases will be searched given the limited history of publication in the field of storytelling in public health.

Medline, Embase, PsycINFO, ERIC, Web of Science, Art and Humanities database (ProQuest), Scopus and Google Scholar will be searched. The reference lists of identified articles will be searched for additional studies, and forward citations of identified articles will be retrieved using University College London libraries. Ongoing or recently completed trials will be searched for using alerts from the above databases.

Grey literature was considered for inclusion but determined unsuitable for this specific review because it aims to focus on studies with well-reported methodologies and findings that have been subject to peer review. Based on the scoping search and given the breadth of topics covered, together with the wide extent of grey literature, as well as the process required to filter and validate the material, inclusion would require a substantially different approach that warrants a separate study.

\section{Search strategy}

This systematic narrative review will identify relevant articles by combining search terms for storytelling; contextcomprising public health issues with a preventative element; change in KAB. The provisional search terms are listed in table 2. English language and dates 1990present will be the only filters.

\section{Study records}

Data management

The search results will be uploaded into reference management software (EndNote) to remove duplicate records of the same report. The unique records will then be uploaded into web-based, systematic review management software (SUMARI; Joanna Briggs Institute, Australia). Using this software, the initial title and abstract screening, and the full-text review will be logged. Both reviewers will use this system. All standardised forms will be piloted and revised as needed by the reviewers before starting the review.

\section{Screening and selection process}

Initial review will be by title only, or title and abstract depending on the quantity of titles returned, and the relevance of information provided.

A scoping search conducted to provide an approximate indication and map of where storytelling has been used in public health research to date found the following quantities of published studies. The search was limited to three databases and five public health topics. Search terms used in the scoping search included storytelling or stories or story; public health; knowledge or attitude* or perception* or behavio*r*. Years 1990 to present were included

\begin{tabular}{|c|c|c|c|}
\hline PICO & Intervention & Context (comparator) & Outcome \\
\hline Concepts & storytelling & Health* & Change* $^{\star}$ \\
\hline \multirow[t]{9}{*}{ Synonyms } & story & 'Climate change' & Attitude* \\
\hline & stories & HIV OR HIV/AIDS & knowledge \\
\hline & & Vaccination OR vaccine* & Behavio? $r^{*}$ \\
\hline & & Cancer adj3 screening & $\begin{array}{l}\text { Perception* } \\
\text { Misperception* } \\
\text { Misinformation* }\end{array}$ \\
\hline & & Obesity OR overweight & Belief $^{*}$ \\
\hline & & Smoking OR 'smoking cessation' & \\
\hline & & 'mental health' OR mental & \\
\hline & & $\begin{array}{l}\text { 'maternal and child health' OR 'mother and child' OR } \\
\text { pregnancy OR 'pregnancy outcomes' }\end{array}$ & \\
\hline & & $\begin{array}{l}\text { 'sexual health' OR ‘sexually transmitted infection”' OR } \\
\text { STI* OR STD* }\end{array}$ & \\
\hline
\end{tabular}

*refers to any expansion of the word to which it refers such that a search will be inclusive 
because storytelling is an emergent research tool and it is unlikely that relevant studies would be published prior to this date.

- Climate change (Scopus 19, Web of Science 8, Medline 3);

- Vaccination (Scopus 9, Medline 8, Web of Science 5);

- Cancer prevention and screening (Scopus 21, Web of Science 5, Medline 13);

- HIV/AIDS (Scopus 31, Web of Science 16, Medline $16)$.

- AMR (no studies were found on storytelling in AMR in this scoping search).

Following the search across public health generally (stage 1), the screening and selection procedure will involve the selection of public health topics as determined by their fit to specified criteria (table 1). Relevant studies will be quantified and a decision made regarding which public health issues and studies (using storytelling as the research tool) to advance to stage 2 which will comprise a full-text review.

In determining the relevance of each of these topics according to the criteria listed in table 1 , vaccination and cancer screening were found to satisfy all listed criteria, followed by climate change and HIV/AIDs. Despite not fulfilling all criteria, the latter two topics would still be included because they fulfil more criteria than not, for example, the only criterion not fulfilled by climate change as a topic is the existence of studies relating to large populations (to date, the use of storytelling in climate change appears to refer to specific populations, eg, Inupuits). However, ultimately findings from this systematic review will inform primary research into the public health issue of AMR and there are enough parallels between the two issues that justify retaining climate change. ${ }^{33}$ HIV/AIDs is an issue that has seen a large amount of community participation in terms of storytelling around the topic. Given the relative paucity of published data on the use of storytelling as a research method, it is proposed that HIV/AIDs is included as a topic of interest in the search. ${ }^{34}$

The criteria in table 1 are addressed by the following questions to be asked of studies identified by stage 1 :

- Is there an individual, immediate cost but a long-term population gain? Does the issue involve an element of prevention?

- Is there misunderstanding, misinformation or misperception associated with the issue?

- Is there a need to change $\mathrm{KAB}$ associated with the issue?

- Which populations are most relevant to this issue? (Ideally, a 'healthy' population in relation to a preventative public health measure).

- Are there publications on storytelling used as a research method for this issue?

The criteria in table 1 were chosen after focusing on the public health issue of particular interest to the researchers, with a view to future research, namely, on the topic of AMR. Although inconclusive, the scoping search did not yield any peer-reviewed primary studies that use storytelling in the field of AMR. Consequently, this protocol proposes that an understanding of the use of storytelling as a tool to gain insight into public KAB might be obtained through focusing on other public health issues that have parallel dimensions to AMR. As such, criteria for the selection of public health issues will be based on characteristics of AMR as a public health issue (as considered in the light of public $\mathrm{KAB}$ ), as well as volume of studies found.

The roots of AMR are multifold; however, of concern to public $\mathrm{KAB}$ in particular are reasons that include the misuse and overuse of antibiotics, whether due to over-prescribing by the clinician, over-demand or misuse by the patient, or over-availability of antibiotics to the public without control measures. Essentially, one means of potentially controlling this misuse might be to improve public perception and behaviours in relation to when and why an antibiotic is needed. Forgoing an antibiotic for a minor infection might entail some personal cost, including a slightly longer illness in the immediate term, but help to reduce the development of antibiotic resistance at a population level in the long term. ${ }^{35}$

Another key feature related to public $\mathrm{KAB}$ around antibiotics is the misunderstanding and misinformation about how resistance arises, as well as how antibiotics should be used. ${ }^{36}$ There is a need among the general public to improve understanding of when and why an antibiotic is needed and how resistance develops. Satisfying this need might involve a greater understanding of public $\mathrm{KAB}$ as well as more effective communication and message dissemination. ${ }^{37} 38$

In light of this, key characteristics of interest include that a personal action and cost now will prevent a widescale, serious public health crisis in the future (eg, AMR or poor rates of cancer detection across a population). Effectively, public health issues with a strong leaning towards preventative health issues will be a preference. Likewise, there will be a preference for issues that, to some extent, rest on a premise of misuse, misconception and misunderstanding by the general public, precipitating a need for change in these elements. Evidence for the use of storytelling as a tool to both understand the issues of concern and effect change is also required.

Due to the scarcity of peer-reviewed and published primary studies on the use of storytelling in the public health issues of interest, studies will be selected if they meet most if not all of the listed criteria.

Each study report will be categorised according to status as 'include', 'exclude' or 'unclear'. Reasons for exclusion of ineligible studies will be recorded, and any uncertainties will be resolved by correspondence with study investigators. Articles categorised as 'include' or 'unclear' will be retrieved, and each will be independently reviewed in full-text format. A second independent reviewer will repeat all stages of the review. In cases of unresolved discrepancy between the two reviewers, a third-party adjudicator will be consulted. 
The study selection process will be recorded and presented in flow diagram format according to the recommendations of PRISMA.

\section{Critical appraisal of study quality}

Critical appraisal of included studies will use the 16-item QATSDD tool developed by Sirriyeh et al for qualitative, quantitative and mixed-methods studies. ${ }^{39}$

There is some debate about the value of critical appraisal of qualitative research due to uncertainty around which criteria should be used to assess a study. This stems from the inherent diversity of data found in qualitative studies, the subjective nature of the data as well as the many different qualitative research methods used. Selection of the most suitable assessment criteria is therefore problematic. ${ }^{40}$ Some researchers argue that weak studies should be excluded, but given the lack of consensus around critical appraisal tools, and the limited range of studies in the field of storytelling in public health research, on full-text review encompassing the extent to which the storytelling method and public health topic are relevant, a decision will be made as to whether to include or exclude a study. If the study comprises low quality, then this will be stated.

The QATSDD tool is designed to provide a score for the body of evidence, which is expressed as a percentage of the maximum possible score. The application of this tool also enables comparisons to be drawn between the qualities of quantitative, qualitative or mixed-methods papers within the same field of research. ${ }^{39}$

\section{Data extraction form}

A standard data extraction form will be customised to serve the purposes of extracting data from qualitative, quantitative and mixed-methods studies.

Data will be extracted by two reviewers and independently entered into the customised form. Disagreement will be resolved by consulting a third review author and uncertainties by correspondence with study investigators.

Information extracted will include (non-exhaustive list)

- Study participants: inclusion and exclusion criteria, method of recruitment/selection and study population characteristics;

- Detail of how the storytelling research tool is applied in the study (either to extract information from participants, or as a tool to effect change in $\mathrm{KAB}$ );

- Study quality and study biases (as per the critical appraisal specified below).

- Insights (including quotations) gained via storytelling that provide information on KAB of study participants. These might be quantitative, qualitative or mixed data.

- Insights (including quotations) on how storytelling can effect change in KAB of study participants. These might be quantitative, qualitative or mixed data.
- Insights that support the validity (or appropriateness or value) of storytelling as a research tool in public health.

- Study funding and conflicts of interest.

Data expected to be sourced from storytelling studies include quotations from stories captured as recounted by tellers of their personal experiences; explanations of digital stories; data on themes identified through analysis of story transcripts (the actual transcripts unlikely to be available); qualitative and quantitative data on changes in knowledge, attitudes and practices/behaviours relating to the storytelling intervention.

\section{Assessment of bias in conducting the systematic review}

The systematic review will be conducted following this prespecified protocol and any differences will be reported between the methods outlined in this protocol and the complete review.

\section{Data synthesis}

Data synthesis will use the narrative synthesis approach developed by the Economic and Social Research Council, as described by Rodgers et al, ${ }^{41}$ that is suitable for qualitative and/or quantitative data. The defining characteristic of narrative synthesis is that it adopts a textual approach to the process of synthesis to 'tell the story' of the findings from the included studies, while it may still include the manipulation of some statistical findings. It can also accommodate questions concerned with the implementation of interventions (ie, the storytelling method or tool), as well as with the effects of interventions in experimental settings (ie, impact of storytelling on KAB) ${ }^{41}$

The narrative synthesis will address the two parts of the research question and effectively comprise two separate syntheses that apply to the use of storytelling: (a) as a tool to understand/gain insight into public KAB and (b) as an intervention to determine the impact (including effect) of storytelling as a tool on changing public KAB. Each respective synthesis will be categorised by the nature of the methodological storytelling approach used, the impact and the validity of storytelling as a research tool as it applies to qualitative data, ${ }^{32}$ the insights obtained via storytelling and the impact in terms of the effectiveness if quantitative measures form part of the study. These categories apply to storytelling as both a tool for extracting information and as a tool to effect change.

Determining the validity of storytelling as a research tool, based on a mixture of qualitative and quantitative data, is challenging. The term 'validity' and its meaning in the context of qualitative research lacks consensus. It tends to mean appropriateness of the tool, processes or data. ${ }^{32}$ One attempt at a definition suggests that validity refers to the integrity and application of the methods undertaken and the precision with which the findings accurately reflect the data. ${ }^{42}$ Noble and Smith suggest an equivalent to the term 'validity' is the term 'truth value'. This recognises that in qualitative data, multiple realities exist; and note that a researcher's personal experiences and viewpoints can have 
an impact on methodological and outcome bias. ${ }^{42}$ Rolfe argues for the recognition that each study is individual and unique, and that the task of producing frameworks and predetermined criteria for assessing the quality of research studies is futile. ${ }^{43}$ In attempting to validate the storytelling studies identified, the findings might be compared with existing $\mathrm{KAB} / \mathrm{KAP}$ (knowledge, attitude and practice) surveys to aid interpretation.

In conducting the narrative synthesis, studies might be viewed as providing data that are framed within the story format and by storytelling. In the context of clinical education, Bleakley ${ }^{14}$ explains that stories can be treated as raw material for narrative inquiry (an analytical approach) or, alternatively, a story as the end product of narrative inquiry (a synthesis approach). The former emphasises the structure of a story by analysing content, and the latter approach emphasises the meaning and social context of a story nurturing a discourse around the meaning of the story.

\section{Proposed value of the systematic review and use of findings}

To gain an understanding based on the systematic review of available peer-reviewed, published studies on the use of storytelling as a research tool to extract information, as well as an intervention to effect change used in a research context in various public health settings that meet the criteria of most, if not all, criteria detailed in table 1.

The findings of this systematic review will have value by potentially informing future research studies into different public health issues, in particular AMR, that employ storytelling as a method to source information or as an intervention to effect change with respect to public KAB.

Contributors The study was conceived by BM. BM developed the eligibility criteria, search strategy, risk of bias assessment strategy and data extraction plan with guidance from AH, LS, CF and MW. BM wrote the manuscript, to which all authors contributed. All contributors meet the ICMJE criteria for authorship.

Funding This work is supported by the Medical Research Foundation (MRF) grant number MRF-145-0004-TPG-AVISO.

Disclaimer The funders played no role in the development of the protocol, in writing of the report or in the decision to submit the protocol for publication.

Competing interests None declared.

Patient consent for publication Not required.

Provenance and peer review Not commissioned; externally peer reviewed.

Open access This is an open access article distributed in accordance with the Creative Commons Attribution Non Commercial (CC BY-NC 4.0) license, which permits others to distribute, remix, adapt, build upon this work non-commercially, and license their derivative works on different terms, provided the original work is properly cited, appropriate credit is given, any changes made indicated, and the use is non-commercial. See: http://creativecommons.org/licenses/by-nc/4.0/.

Author note Research question: What evidence supports the use of storytelling as a research tool: (a) to gain insight into public knowledge, attitudes and behaviour $(K A B)$, and (b) as an intervention to effect change in public $K A B$, in relation to issues of public health?

\section{REFERENCES}

1 Wilson M. Some thoughts on storytelling, science and dealing with a post-truth world; 2018.

2 Merleau-Ponty M, Edie JM. The primacy of perception: and other essays on phenomenological psychology, the philosophy of art, history, and politics, 1964: 228.
3 Mead G. Storytelling. In: The SAGE encyclopedia of action research, 2014.

4 Hartling L, Scott SD, Johnson DW, et al. A randomized controlled trial of storytelling as a communication tool. PLoS One 2013;8:e77800-11.

5 Moezzi M, Janda KB, Rotmann S. Using stories, narratives, and storytelling in energy and climate change research. Energy Res Soc Sci 2017;31:1-10.

6 Beemgee. Story vs. narrative. Available: https://www.beemgee.com/ blog/story-vs-narrative/ [Accessed 15 Jan 2019].

7 Paschen J-A, Ison R. Narrative research in climate change adaptation-exploring a complementary paradigm for research and governance. Res Policy 2014;43:1083-92.

8 Williams-Brown S, Baldwin DM, Bakos A. Storytelling as a method to teach African American women breast health information. J Cancer Educ 2002;17.

9 The challenges of being an insider in storytelling research. Available: www.nurseresearcher.co.uk [Accessed 12 Aug 2019].

10 Lenette C, Cox L, Brough M. Digital storytelling as a social work tool: learning from ethnographic research with women from refugee backgrounds. Br J Soc Work 2015;45:988-1005.

11 Taylor B. Hospice nurses tell their stories about a good death: the value of storytelling as a qualitative health research method. Annu Rev Health Soc Sci 1993;3:97-108.

12 Bird S, Wiles JL, Okalik L, et al. Methodological consideration of story telling in qualitative research involving Indigenous peoples. Glob Health Promot 2009;16:16-26.

13 McNamara P. Feminist ethnography. Qualitative Social Work 2009;8:161-77.

14 Bleakley A. Stories as data, data as stories: making sense of narrative inquiry in clinical education. Med Educ 2005;39:534-40.

15 Columbia University Irving Medical Center. Columbia narrative medicine, 2019. Available: https://www.narrativemedicine.org/aboutnarrative-medicine/. 2019

16 Charon R. Narrative medicine. JAMA 2001;286.

17 Cueva M, Kuhnley R, Slatton J, et al. Telenovela: an innovative colorectal cancer screening health messaging tool. Int J Circumpolar Health 2013;72:21301.

18 Otto D. Lived experience of climate change-a digital storytelling approach. IJGW 2017;12.

19 Limaye NP, Rivas-Nieto AC, Carcamo CP, et al. Nuestras Historias designing a novel digital story intervention through participatory methods to improve maternal and child health in the Peruvian Amazon. PLoS One 2018;13:e0205673-22.

20 Hopfer S, Garcia S, Duong HT, et al. A narrative engagement framework to understand HPV vaccination among Latina and Vietnamese women in a planned parenthood setting. Health Educ Behav 2017;44:738-47.

21 Rieger KL, West CH, Kenny A, et al. Digital storytelling as a method in health research: a systematic review protocol. Syst Rev 2018;7:41.

22 Dahlstrom MF. Using narratives and storytelling to communicate science with nonexpert audiences. Proc Natl Acad Sci U S A 2014;111 Suppl 4:13614-20.

23 Cherrington A, Williams JH, Foster PP, et al. Narratives to enhance smoking cessation interventions among African-American smokers, the ACCE project. BMC Res Notes 2015;8:567.

24 Scott SD, Brett-MacLean P, Archibald M, et al. Protocol for a systematic review of the use of narrative storytelling and visual-artsbased approaches as knowledge translation tools in healthcare. Syst Rev 2013;2:19.

25 Hinyard LJ, Kreuter MW. Using narrative communication as a tool for health behavior change: a conceptual, theoretical, and empirical overview. Health Educ Behav 2007;34:777-92.

26 Stemming the Superbug Tide-Just A Few Dollars More, 2018. Available: https://doi.org/10.1787/9789264307599-en

27 Edgar T, Boyd SD, Palamé MJ. Sustainability for behaviour change in the fight against antibiotic resistance: a social marketing framework. $J$ Antimicrob Chemother 2009;63:230-7.

28 Hawkings NJ, Wood F, Butler CC. Public attitudes towards bacterial resistance: a qualitative study. J Antimicrob Chemother 2007;59:1155-60.

29 Van Hecke O, Butler CC, Wang K, et al. Parents' perceptions of antibiotic use and antibiotic resistance (pause): a qualitative interview study. J Antimicrob Chemother 2019;74:1741-7.

30 Shamseer L, Moher D, Clarke M, et al. Preferred reporting items for systematic review and meta-analysis protocols (PRISMA-P) 2015: elaboration and explanation. BMJ 2015;349.

31 HMSO, Great Britain. Public health in England: the report of the Committee of Inquiry into the Future Development of the Public Health Function. London: H.M.S.O, 1988. 
32 Leung L. Validity, reliability, and generalizability in qualitative research. J Family Med Prim Care 2015;4.

33 The Lancet Respiratory Medicine. Antimicrobial resistance-what can we learn from climate change? Lancet Respir Med 2016;4.

34 Treffry-Goatley A, Lessells RJ, Moletsane R, et al. Community engagement with HIV drug adherence in rural South Africa: a transdisciplinary approach. Med Humanit 2018;44:239-46.

35 CDDEP. CDDEP: The Center for Disease Dynamics E, Policy. The State of the World's Antibiotics, 2015. Available: www.cddep.org [Accessed 12 Mar 2019].

36 Exploring the consumer perspective on antimicrobial resistance, 2015. Available: https://wellcome.ac.uk/sites/default/files/exploringconsumer-perspective-on-antimicrobial-resistance-jun15.pdf [Accessed 26 Nov 2018].

37 Mcnulty CAM, Nichols T, Boyle PJ, et al. The English antibiotic awareness campaigns: did they change the public's knowledge of and attitudes to antibiotic use? Available: http://jac.oxfordjournals. org/ [Accessed 26 Nov 2018].

38 McNulty CAM, Boyle P, Nichols T, et al. The public's attitudes to and compliance with antibiotics. J Antimicrob Chemother 2007;60 Suppl $1: i 63-8$.

39 Sirriyeh R, Lawton R, Gardner P, et al. Reviewing studies with diverse designs: the development and evaluation of a new tool. J Eval Clin Pract 2012;18:746-52.

40 Mary D-W, Dixon-Woods M, Bonas S, et al. How can systematic reviews incorporate qualitative research? A critical perspective. Qualitative Research 2006;6:27-44.

41 Rodgers M, Arai L, Britten N, et al. Guidance on the conduct of narrative synthesis in systematic reviews: a comparison of guidanceled narrative synthesis versus meta-analysis. A product from the ESRC Methods Programme, 2006: 1-92.

42 Noble H, Smith J. Issues of validity and reliability in qualitative research. Evid Based Nurs 2015;18:34-5

43 Rolfe G. Validity, trustworthiness and rigour: quality and the idea of qualitative research. J Adv Nurs 2006;53:304-10.

44 Smith DRM, Dolk FCK, Pouwels KB, et al. Defining the appropriateness and inappropriateness of antibiotic prescribing in primary care. J Antimicrob Chemother 2018;73:ii11-18.

45 Mason T, Trochez C, Thomas R, et al. Knowledge and awareness of the general public and perception of pharmacists about antibiotic resistance. BMC Public Health 2018;18:711.

46 Hay AD, Heron J, Ness A, et al. The prevalence of symptoms and consultations in pre-school children in the Avon Longitudinal Study of Parents and Children (ALSPAC): a prospective cohort study. Fam Pract 2005;22:367-74
47 Thompson PL, Spyridis N, Sharland M, et al. Changes in clinical indications for community antibiotic prescribing for children in the UK from 1996 to 2006: will the new NICE prescribing guidance on upper respiratory tract infections just be ignored? Arch Dis Child 1996;94:337-40.

48 McGregor LM, von Wagner C, Vart G, et al. The impact of supplementary narrative-based information on colorectal cancer screening beliefs and intention. BMC Cancer 2015;15.

49 Larkey LK, Gonzalez J. Storytelling for promoting colorectal cancer prevention and early detection among Latinos. Patient Educ Couns 2007;67:272-8.

50 Larkey LK, Lopez AM, Minnal A, et al. Storytelling for promoting colorectal cancer screening among underserved Latina women: a randomized pilot study. Cancer Control 2009;16:79-87.

51 Raithatha N, Holland R, Gerrard S, et al. A qualitative investigation of vaccine risk perception amongst parents who immunize their children: a matter of public health concern. J Public Health Med 2003;25:161-4.

52 Lee H, Kim D, Kiang PN-chu, et al. Awareness, knowledge, social norms, and vaccination intentions among Khmer mother-daughter pairs. Ethn Health 2018;21:1-13.

53 Shelby A, Ernst K. Story and science: how providers and parents can utilize storytelling to combat anti-vaccine misinformation. Hum Vaccin Immunother 2013;9:1795-801.

54 Capstick SB, Pidgeon NF. What is climate change scepticism? Examination of the concept using a mixed methods study of the UK public. Global Environmental Change

55 Tobler C, Visschers VHM, Siegrist M. Addressing climate change: determinants of consumers' willingness to act and to support policy measures. J Environ Psychol 2012;32:197-207.

56 Taylor AL, Dessai S, Bruine de Bruin W. Public perception of climate risk and adaptation in the UK: a review of the literature. Clim Risk Manag 2014;4-5:1-16.

57 Harper SL, Edge VL, Cunsolo Willox A, Willox AC, et al. 'Changing climate, changing health, changing stories' profile: using an EcoHealth approach to explore impacts of climate change on Inuit health. Ecohealth 2012;9:89-101.

58 Cunsolo Willox A, Harper SL, Edge VL, et al. Storytelling in a digital age: digital storytelling as an emerging narrative method for preserving and promoting Indigenous oral wisdom. Qualitative Research 2013;13:127-47.

59 Sakakibara C. "Our Home Is Drowning": Iñupiat storytelling and climate change in Point Hope, Alaska. Geogr Rev 2008;98:456-75. 